\title{
Reasoning with conditionals: Does every counterexample count? It's frequency that counts
}

\author{
SONJA M. Geiger \\ University of Potsdam, Potsdam, Germany \\ AND \\ KLaus Oberauer \\ University of Bristol, Bristol, England
}

\begin{abstract}
A series of experiments investigated what determines people's degree of belief in conditionals and their readiness to draw inferences from them. Information on the frequency of exceptions to conditional rules was contrasted with information about the number of different disabling conditions causing these exceptions. Experiments 1 and 2, using conditionals with arbitrary contents, revealed a strong effect of frequency information and no effect of disabling information. Experiment 3 established that, in the absence of frequency information, the disabling condition information used in Experiments 1 and 2 affected belief in the conditionals and inference acceptance, as has been found in many previous studies (Byrne, 1989; DeNeys, Schaeken, \& d'Ydewalle, 2003b). Experiment 4 extended the results of Experiments 1 and 2 to everyday conditionals. The results show that belief in a conditional, and the confidence in inferences subsequently drawn from it, both depend on the subjective conditional probability of the consequent given the antecedent. This probability is estimated from the relative frequency of exceptions regardless of what causes them.
\end{abstract}

"Exceptions confirm the rule," says a German proverb. The view expressed by it summarizes people's everyday experience that nothing in this world is certain and that belief in a general rule should not be completely abandoned in the face of exceptions that show up every now and then. In research on reasoning with conditional statements such as if $p$ then $q$, a probabilistic perspective has been advanced over the last 10 years (see, e.g., Anderson, 1995; Evans \& Over, 2004; Oaksford \& Chater, 1994, 2001). According to this view, people hold a degree of belief in a conditional statement that depends on the subjective conditional probability of the consequent given the antecedent. Some researchers pursuing the probabilistic approach assume that people evaluate their belief in a conditional rule according to a procedure called the Ramsey test (Evans \& Over, 2004 , ch. 2). When evaluating the belief in a rule such as "if you open the fridge, then a light inside goes on," people thus suppose $p$ in a mental simulation (in which the fridge door is opened) and then relate the number of $p q$ cases to the number of $p \neg q$ cases (" $\neg$ " denotes the negation of $q$ ) within this suppositional framework (Evans, Over, \& Handley, 2005; Over \& Evans, 2003). This comes down to comparing rule-confirming cases (in which the fridge was opened and the light went on) with exceptions (in which the fridge was opened and the light did not go on). The higher this ratio is - that is, the fewer exceptions to confirming cases there are - the higher people's belief in the conditional rule will be.
Extensive testing of this idea was first provided by Evans, Handley, and Over (2003) and Oberauer and Wilhelm (2003). Both research groups independently designed a task that has come to be known as the probabilistic truth table task. In this task, participants are provided with a conditional statement and explicit frequency information about the four cases that comprise the conditional's truth table - the conjunctions of $p q, p \neg q, \neg p q$, and $\neg p \neg q$. Participants are then asked to evaluate their belief in the conditional statement considering the frequency information given. In several studies (Evans et al., 2003; Oberauer, Geiger, Fischer, \& Weidenfeld, 2007; Oberauer \& Wilhelm, 2003), the conditional probability $P(q \mid p)$ had the largest influence on people's belief judgments.

If conditionals allow for exceptions, inferences from conditionals must have a degree of uncertainty. Research on reasoning with conditional statements as premises corroborates this assumption. A large body of research has investigated the role of counterexamples on inferences drawn from conditional statements (see, e.g., Byrne, 1989; Cummins, 1995; De Neys, Schaeken, \& d'Ydewalle, 2003b; Markovits \& Potvin, 2001). Byrne and colleagues (Byrne, 1989; Byrne, Espino, \& Santamaria, 1999) were among the first researchers to systematically investigate effects of counterexamples on inference tasks such as modus ponens (MP) and modus tollens (MT). In several experiments, Byrne and colleagues showed that if a conditional rule was accompanied by an additional necessary

S. M. Geiger, geiger@cyllene.uwa.edu.au 
precondition, the acceptance of otherwise valid inferences declined. For example, if people were presented with the statements

$$
\begin{aligned}
& \text { If you open the fridge, then the light inside will go } \\
& \text { on. } \\
& \text { If the light bulb is working, then the light inside will } \\
& \text { go on. }
\end{aligned}
$$$$
\text { Somebody opens the fridge. }
$$

they tended to reject the consequent the light inside will go on (example of an MP inference) more often when the second antecedent was present (If the light bulb is working) than when it was omitted. Byrne explains this so-called MP suppression effect with the enhanced availability of counterexamples for the conditional. In our interpretation, the disabling condition of a broken bulb leads to the $p \neg q$ situation (open door, dark fridge) in defiance of MP.

The suppression effect of counterexamples has since been widely replicated (Cummins, 1995; Cummins, Lubart, Alksnis, \& Rist, 1991; Markovits \& Potvin, 2001; Thompson, 1995). For instance, Cummins showed that the suppression effect also prevailed if disabling conditions were not explicitly mentioned but could be retrieved from everyday experience. De Neys et al. (2003b) demonstrated that the retrieval of counterexamples was a continuous process in which every additional counterexample lowered the acceptance of inferences. A shared feature of these studies on inference suppression is that the counterexamples that they gave to or asked from participants were in the form of conditions (Weidenfeld, Oberauer, \& Hörnig, 2005), reasons, explanations (Verschueren, Schaeken, \& d'Ydewalle, 2005), or factors (Cummins, 1995; De Neys, Schaeken, \& d'Ydewalle, 2002, 2003b) that allowed or caused exceptions to occur. For example, Cummins (1995) asked participants to "please write down as many factors [italics added] as you can that could make this situation (the $p \neg q$ case) possible (p. 651).” The number of different disabling conditions needs to be distinguished from the number of exceptional instances. For example, there may be just one disabling condition that can cause a fridge to stay dark when the door is opened (i.e., a broken light bulb), but the frequency of this occurrence may be high when the light bulbs used in fridges are unreliable. We will call an individual instance of $p \neg q$ an exception and a potential cause leading to the occurrence of one or many such exceptions a disabling condition.

So far, both lines of research - on the probability of conditionals and on the suppression of logically valid inferences - have confirmed an important role for the $p \neg q$ case in reducing the confidence people put in conditionals and in the inferences they draw from them. However, these lines of research have mainly used one of two types of information to investigate the $p \neg q$ cases - frequency information about such cases in work on the probability of conditionals and counterexample information relating to (causal) schemata stored in semantic memory in work on the suppression of logically valid inferences. A researcher who is explicitly investigating the effects of information about disabling conditions and the frequency of occur- rences of $p \neg q$ cases in conditional reasoning is Verschueren (Verschueren et al., 2005). In her dual process account of everyday conditional reasoning, Verschueren differentiates two kinds of information: probability information and counterexample information. She conceptualizes the former as information stored in long-term memory about the frequency of instances, which serves as input for a fast, heuristic probability estimation process that allows gradual responses. Counterexample information about disabling conditions is described as general knowledge about the causal relations of events - for instance, knowledge that broken bulbs do not emit light. This knowledge has to be retrieved in a more effortful way than does probability information and thus leads to slower judgments.

In their study, Verschueren et al. (2005) obtained probability ratings and disabler ratings for everyday conditionals such as If you water a plant well, then it will stay green. For the probability ratings, participants were asked "A plant is well watered; how likely is it that it will stay green?" and had to choose among never, almost never, sometimes, most of the time, and always. Ratings of these frequency categories were taken as subjective probability estimates. For the disabling conditions, participants were asked questions such as Can a plant be well watered and not stay green? If yes, list one reason. Another group of participants evaluated inferences derived from these conditionals. Results confirmed the dual process assumption: People who mainly relied on disabling information took longer to evaluate MP and MT, whereas people who mainly relied on subjective probabilities took less time.

A weakness of Verschueren et al.'s (2005) study is that the two types of information are usually highly correlated for everyday conditionals: The more different causes there are to prevent $q$ despite the presence of $p$, the more frequent the instances of $p \neg q$ will usually be. For any given inference evaluation supposedly based on frequency information, it can never be ruled out that people arrived at their answer by thinking of disabling conditions.

The present study has two goals: first, to explicitly contrast the two types of information that have so far been used mostly in separate studies, albeit in related fields, while avoiding the potential confound existing in the Verschueren et al. (2005) study; second, to integrate the hitherto mainly unrelated findings on the believability of the conditional obtained using the probabilistic truth table task with the findings on the suppression effect on acceptance of inferences. To our knowledge, only a few studies have investigated the effect of disabling conditions on the interpretation of the conditional (see, e.g., Dieussaert, Schaeken, \& d'Ydewalle, 2002) or the effect of frequency manipulations of exceptions on inference tasks (Geiger \& Oberauer, 2006; Oaksford, Chater, \& Larkin, 2000). To close this gap, we measured people's beliefs in a conditional and their willingness to derive MP and MT inferences from it, while we manipulated both types of information.

This approach allowed us to test four alternative hypotheses. Hypothesis 1 follows from the probabilistic approach, which predicts that people's belief in a conditional depends on the relative frequency of situations in which both the antecedent and the consequent are true, out of all 
situations in which the antecedent is true - that is, $P(q \mid p)$. Thus, Hypothesis 1 is that people's confidence in an inference drawn from a conditional depends on their belief in this conditional (Evans \& Over, 2004) and hence is influenced by the same frequency information.

Hypothesis 2 represents the mental model account, which in its strong form predicts that the retrieval of one disabling condition blocks inferences from a conditional, regardless of how few exceptions are caused by this condition. Against this assumption, De Neys et al. (2003b) have shown that people's acceptability ratings of MP and MT inferences gradually decline with the number of disabling conditions. It cannot be ruled out, however, that when the major premise of an inference problem is a conditional with many disabling conditions, people are simply more likely to retrieve any one of them, leading them to reject the inference. De Neys et al. (2003b) also considered an extension of the mental model theory in which each disabling condition was represented as a separate model. This modified mental model theory would therefore predict an effect of the number of disabling conditions on inference acceptance ratings. We see no role for frequency information in an inference procedure based on mental models; thus, even the modified mental model account should not predict an effect of the frequency of exceptions.

According to Hypothesis 3, both frequency information and information about disabling conditions could influence the believability of the conditional and the acceptance of inferences. Evidence for this prediction comes from Weidenfeld et al. (2005) and Verschueren et al. (2005). According to their dual process assumptions, we should expect additive effects of both variables.

Hypothesis 4 assumes that solving the two types of tasks - evaluating the believability of a conditional and evaluating inferences drawn from it-relies on different sources of information. On the basis of the research reported above, it seems justified to speculate that the analytic nature of drawing inferences leads people to draw more heavily on information about disabling conditions, whereas the estimation of the believability of a conditional invites people to give probability estimates on the basis of frequencies. If this is correct, the bulk of the research reviewed above was intuitively justified in testing only one side of things. Hypothesis 4 predicts that people's judgment of the probability of a conditional depends on the frequency of exceptions but not on the number of disabling conditions; the reverse pattern should be observed for acceptance of MP and MT inferences. Task-dependent selection and processing of information have been shown for different reasoning tasks with conditionals before (Thompson, 2000) and thus do not seem unlikely.

To distinguish between these four hypotheses, in Experiment 1 , we combined a probabilistic truth table task that gave explicit frequencies of truth table cases (Oberauer \& Wilhelm, 2003) with a suppression paradigm that presented additional disabling conditions. Experiment 2 replicated that design with a reduced array of frequency information. Experiment 3 tested whether the frequently shown suppression effect could be replicated with disabling condition information in the absence of frequency information. Experiment 4 extended the findings to conditionals with everyday content, for which the two critical information dimensions were measured by independent ratings. We asked participants to evaluate the believability of the conditional and all four basic inferences from the following conditional premises: MP, MT, acceptance of the consequent (AC), and denial of the antecedent (DA). On the basis of theoretical assumptions and previous studies, we expected the manipulations of exception frequencies and numbers of disablers to affect only MP and MT; endorsement of DA and AC depend on another kind of counterexample called alternative causes, which were not investigated here (for effects of alternative causes on AC and DA, see Cummins, 1995; Thompson, 1995, 2000; Verschueren et al., 2005).

\section{EXPERIMENT 1}

In Experiment 1, we manipulated information about the number of exceptional cases and disabling conditions independently. Exceptional cases were introduced through explicit information about the frequencies of the conjunction of $p \neg q$ cases, as well as the conditional truth table's three other cases, the conjunctions of $p q, \neg p q$, and $\neg p \neg q$. Different cover stories introduced a conditional statement concerning 2,000 cases of a given population, which were distributed over the four truth table cases in varying frequencies. Disabling conditions were given in the form of a conditional sentence that introduced a circumstance in which $\neg q$ was possible even though $p$ was true (De Neys et al., 2003b). The frequencies of exceptions and the numbers of disablers assigned in each condition are summarized in Table 1 . We refer to the four conditions as FF, FM, MF, and MM, with the first letter referring to few or many exceptions and the second letter referring to few or many disablers. The conditions with few disablers actually had zero disablers; for the conditions with many, we used three disablers, following De Neys et al. (2003b), who showed that the availability of at least three disabling conditions had a reasonably large effect on the acceptance of inference tasks.

\section{Method}

Participants. Twenty-seven first-year psychology students (age range: $19-30$ years) from the University of Potsdam participated. The

Table 1 Design Used in Experiments 1 and 2

\begin{tabular}{cccccc}
\hline & \multicolumn{2}{c}{ Experiment 1} & & \multicolumn{2}{c}{ Experiment 2} \\
\cline { 2 - 3 } Category & $\begin{array}{l}\text { Ratio of } \\
p \neg q \text { Cases } \\
\text { to } p \text { Cases }\end{array}$ & $\begin{array}{c}\text { No. Disabling } \\
\text { Conditions }\end{array}$ & & $\begin{array}{l}\text { Ratio of } \\
p \neg q \text { Cases } \\
\text { to } p \text { Cases }\end{array}$ & $\begin{array}{c}\text { No. Disabling } \\
\text { Conditions }\end{array}$ \\
\hline FF & $100: 1,000$ & 0 & $150: 1,000$ & 1 \\
FM & $100: 1,000$ & 3 & & $150: 1,000$ & 3 \\
MF & $900: 1,800$ & 0 & $450: 1,000$ & 1 \\
MM & $900: 1,800$ & 3 & $450: 1,000$ & 3 \\
\hline
\end{tabular}

Note-FF, few single exceptions, few (in Experiment 1, zero) disabling conditions; FM, few single exceptions, many (three) disabling conditions; MF, many single exceptions, few (in Experiment 1, zero) disabling conditions; MM, many single exceptions, many (three) disabling conditions. 
order of tasks was varied among participants; one group of 14 first made probability judgments and then solved reasoning tasks, and the other 13 participants performed these tasks in the opposite order.

Materials and Procedure. The experiment was a withinsubjects study that followed the design presented in Table 1. For the probability judgments, the participants read eight fictional short stories that were presented on a computer monitor. Each story required participants to complete a judgment task; participants thus completed eight tasks, two for each condition listed in Table 1. The stories were set on a foreign planet called Noxus, and related invented scientific information about the planet. The cover stories were inspired by Cummins (1995), who used similar pseudonatural material in her second experiment to prevent people from retrieving disabling conditions from their world knowledge. In addition to using Cummins's story, we used seven more cover stories of our own invention. Each cover story included a conditional statement made by an "expert." Preceding the conditional statement, either zero or three disabling conditions were listed for it. Additionally, 2,000 cases involving life on Noxus, including a given number of $p q$ cases, were listed, as shown in Table 1. Here is an example of a cover story (for the MM condition). (For a list of all the conditional statements and their disabling conditions used in Experiments 1-3, see Appendix A.)

A team of biologists examines the different species on Noxus. They focus on genetic relationships expressed by body characteristics, such as the number of legs and the shape of ears. They find that if an animal belongs to the family of grocks, then it has six legs.

It is also known that:

- If a grock has a genetic mutation, then it has fewer than six legs.

- If a grock is born to lop-eared parents, then it has fewer than six legs.

- If a grock has run into a trap for rat-like animals, then it has fewer than six legs.

Of 2,000 animals that have been examined over the last six months, scientists have made the following records:

900 animals that belonged to the family of grocks had six legs.

900 animals that did not belong to the family of grocks did not have six legs.

100 animals that did not belong to the family of grocks had six legs.
100 animals that did not belong to the family of grocks did not have six legs.

According to a Noxus expert, "If an animal belongs to the family of grocks, then it has six legs."

Participants were asked to rate the probability that the expert was right on a scale from 0 (absolutely impossible) to 100 (absolutely certain).

Either before or after the probability judgment of conditionals portion of the experiment, participants had to solve the four types of inference tasks for each cover story. The order of the cover stories and conditions was randomized in the same manner as the probability judgment part of the experiment. The cover stories and conditionals were presented again, this time followed by MP, MT, DA, and AC tasks on one specific animal drawn from the sample. Order of the inference tasks was randomized anew for each cover story.

Here is an example of an inference task for MP:

Expert's statement: "If the animal belongs to the family of grocks, then it has six legs."

Observation: The animal belongs to the family of grocks.

Conclusion: It has six legs.

Participants had to rate their confidence in the conclusion on a six-point scale ranging from certain that I can draw the conclusion to certain that I cannot draw the conclusion.

\section{Results}

Results from Experiment 1 on the believability judgments of the conditional sentences and the acceptance of the four inference forms are summarized in Table 2. Since the task order factor did not have a general effect and did not interact with any of the other factors, all data were collapsed across this factor and submitted to a 2 (number of exceptional $p q$ cases: few vs. many) $\times 2$ (number of disabling conditions: few vs. many) ANOVA. In all of the experiments in the present study, answers for the inference tasks were linearly transformed to fit a scale from +5 (certain that I can draw this conclusion) to -5 (certain that I cannot draw this conclusion) in steps of two. This rescaling makes our data comparable to data from previous research that used the same scale (Cummins et al., 1991; De Neys et al., 2003b; Oaksford et al., 2000) and has the advantage of indicating a rejection of the conclusion with negative values and an acceptance of the conclusion with

Table 2

Results of Experiments 1 and 2

\begin{tabular}{|c|c|c|c|c|c|c|c|c|c|c|c|c|c|c|c|c|}
\hline \multirow{3}{*}{$\begin{array}{c}\text { Dependent } \\
\text { Variable }\end{array}$} & \multicolumn{8}{|c|}{ Experiment 1} & \multicolumn{8}{|c|}{ Experiment 2} \\
\hline & \multicolumn{2}{|c|}{ FF } & \multicolumn{2}{|c|}{ FM } & \multicolumn{2}{|c|}{ MF } & \multicolumn{2}{|c|}{ MM } & \multicolumn{2}{|c|}{ FF } & \multicolumn{2}{|c|}{ FM } & \multicolumn{2}{|c|}{ MF } & \multicolumn{2}{|c|}{ MM } \\
\hline & $M$ & $S D$ & $M$ & $S D$ & $M$ & $S D$ & $M$ & $S D$ & $M$ & $S D$ & $M$ & $S D$ & $M$ & $S D$ & $M$ & $S D$ \\
\hline (conditiona & 74.1 & 19.3 & 73.1 & 20.8 & 54.5 & 13.4 & 51.3 & 15. & 73.8 & 22.4 & 70.9 & 23.8 & 55.4 & 9.9 & 57.5 & 11.0 \\
\hline AP & 1.9 & 2.4 & 1. & 2.7 & 0 & 3.1 & -0.7 & 2 & 1.2 & 3 & 0.7 & 2.9 & 0.0 & 2.6 & -0.5 & 2.8 \\
\hline MT & 0.3 & 2.3 & 0.2 & 2.2 & -1.2 & 2.3 & -0.9 & 2.6 & -1.1 & 2.9 & 0.0 & 2.6 & -1.1 & 2.3 & -0.7 & 2.3 \\
\hline $\mathrm{AC}$ & 0.4 & 2.7 & 0.1 & 2.5 & 0.6 & 2.2 & 0.1 & 2.8 & 2.3 & 2.4 & 1.3 & 2.7 & 1.5 & 2.3 & 1.3 & 2.4 \\
\hline DA & -1.2 & 1.9 & -1.3 & 1.9 & -1.1 & 2.1 & -1.0 & 2.1 & 1.2 & 2.6 & 0.7 & 2.4 & 0.7 & 2.7 & 1.4 & 2.3 \\
\hline
\end{tabular}

Note- $P$ (conditional), probability of the conditional. Estimates for this variable were given on a scale from 0 to 100 . Estimates for the inference tasks could range from -5 to +5 . FF, few single exceptions, few (or no) disabling conditions; FM, few single exceptions, many (three) disabling conditions; MF, many single exceptions, few (or no) disabling conditions; MM, many single exceptions, many (three) disabling conditions. MP, modus ponens; MT, modus tollens; AC, acceptance of the consequent; DA, denial of the antecedent. 
positive values. Since the midpoint of uncertainty was not represented on the answer scale (cf. Oaksford et al., 2000, Experiments 2 and 3), the steps of two ensured equal numerical distances between the six answer options.

The number of exceptional cases had an effect on the probability ratings of the conditional $P($ cond $)[F(1,26)=$ $\left.23.1, p<.001, \eta_{\mathrm{p}}^{2}=0.47\right]$ and on MP $[F(1,26)=34.0$, $\left.p<.001, \eta_{\mathrm{p}}^{2}=0.50\right]$, and a somewhat smaller effect on MT $\left[F(1,26)=17.0, p<.001, \eta_{\mathrm{p}}^{2}=0.39\right]$. For MP, there was an interaction between the number of exceptions and disabling conditions $\left[F(1,26)=3.8, p=.06, \eta_{\mathrm{p}}^{2}=\right.$ $0.13]$. None of the other effects reached significance (all $\left.F_{\mathrm{s}}<1\right)$. For AC and DA, neither of the two types of information had an effect (all $F \mathrm{~s}<2$ ).

\section{Discussion}

The number of disabling conditions had no effect on any of the dependent variables when additional frequency information was available. The suppression effect of disabling conditions on the inference tasks MP and MT is a very well-established effect (Byrne et al., 1999; Cummins, 1995; De Neys et al., 2003b; Johnson-Laird, 2001; Quinn \& Markovits, 1998), and the availability of disabling conditions has also been shown to reduce the perceived sufficiency of a conditional (Dieussaert et al., 2002) and, consequently, its perceived probability (Weidenfeld et al., 2005). In this experiment, the effects of the availability of disabling conditions seem to have been superseded by the processing of frequency information. The notion that the availability of frequency information renders the adherence to disabling information unnecessary has been raised by Weidenfeld (2004), who also found that the availability of disabling conditions, when combined with frequency information, no longer affected MP and MT.

One reason for the lack of disabling condition effects may be the way in which the information was presented. The material in Experiment 1 stated the disabling conditions as additional information, unrelated to the frequency of $p q$ cases in question. To address the issue of whether the effect of higher order disabling information would prevail if it were made superordinate to frequency information about single exceptions, we conducted a second experiment, introducing some minor changes.

\section{EXPERIMENT 2}

In Experiment 2, we used a reduced version of the probabilistic truth table task. Instead of giving frequency information about all truth table cases, only the overall number of true antecedent cases $(1,000)$ was stated, and the number of exceptions ( $p q$ cases) was directly attributed to either one or three categories of disabling conditions. In this way, the role of disabling conditions was emphasized as causing (or enabling) a certain number of exceptions, whereas overall frequency information about the exceptions had to be indirectly inferred (by adding all $p q$ cases attributed to the different disabling conditions and relating them to all $p$ cases). The number of exceptions was slightly changed, from 100 and 900 exceptional cases in the FF/FM and MF/ MM conditions, respectively, in Experiment 1, to 150 and
450 exceptional cases in the FF/FM and MF/MM conditions, respectively, in Experiment 2; this was done to make the number of such cases easily divisible by three and to keep $P(q \mid p)$ reasonably close to 0.5 for the many exceptions condition (see Table 1). The number of disabling conditions in the few exceptions condition was changed from zero (in Experiment 1) to one, since the existing exceptions were always attributed to some reason. In the condition with only one category of disabling conditions, this category accounted for all (150 FF/FM and $450 \mathrm{MF} /$ MM) exceptions; in the condition with three categories of disabling conditions, each category accounted for a third of the exceptions - 50 in the FF/FM conditions and 150 in the MF/MM conditions.

The different disabling conditions can be regarded as categories for the exceptions that they cause, and therefore, the four conditions can be interpreted as two packed conditions (one reason for all exceptions) versus two unpacked conditions (three reasons for the same number of exceptions). The notion of packed versus unpacked descriptions for the same probability was first introduced by Tversky and collaborators in their support theory (Rottenstreich \& Tversky, 1997; Tversky \& Koehler, 1994). According to the support theory, subjective probability judgments should be higher for a description of a situation that is broken down into more specific categories (that is, unpacked). In the present experiments, it is the probability of exceptions that is unpacked into three different categories. If this probability is judged higher in the conditions with unpacked causes, the ratings for the believability judgments for the conditionals and acceptance of the inferences should be lower in these conditions. Tversky and collaborators would therefore predict that the number of disabling conditions should have an effect, even if frequency information is simultaneously available and held constant: The more different causes for the same number of exceptional cases that are presented, the lower the believability ratings for the conditional and acceptance of inferences should be.

\section{Method}

Participants. Thirty students (age range: $20-31$ years) from the University of Potsdam, majoring in various subjects, participated in this experiment. The order of tasks was varied among participants; one group of 15 first made probability judgments and then solved reasoning tasks, and the other 15 participants performed these tasks in the opposite order.

Materials and Procedure. The materials and procedure used were exactly the same as those used in Experiment 1, except for the minor changes in the presented material mentioned previously.

Here is an example of a cover story (for the MM condition).

A team of biologists examines the different species on Noxus. They focus on genetic relationships such as number of legs and the shape of ears. They find that if an animal belongs to the family of grocks, then it has six legs.

Of 1,000 animals of the family of grocks that have been examined, it is also known that:

150 of these grocks did not have six legs because of a genetic mutation. 
150 of these grocks did not have six legs because they had lopeared parents.

150 of these grocks did not have six legs because they ran into a trap for rat-like animals.

The presentation of the probability judgment questions and the four inference tasks was identical to that of Experiment 1 in form and order.

\section{Results}

Since the task order factor did not have a general effect and did not interact with any of the other factors, all data were collapsed across this factor and submitted to a 2 (number of exceptional $[p \neg q]$ cases: few vs. many) $\times 2$ (number of disabling conditions: few vs. many) ANOVA. The number of exceptions had an effect on the believability ratings of the conditional $P($ cond $)[F(1,29)=26.8$, $\left.p<.001, \eta_{\mathrm{p}}^{2}=0.48\right]$ and on MP $[F(1,29)=10.3, p<$ $\left..01, \eta_{\mathrm{p}}^{2}=0.26\right]$. The number of disabling conditions had a small effect on MT $\left[F(1,29)=4.6, p<.05, \eta_{\mathrm{p}}^{2}=0.14\right]$. Contrary to general findings on the suppression effect, a higher number of disabling conditions led to a higher acceptance of MT. None of the other effects, including those on AC and DA, reached significance (all $F \mathrm{~s}<2.1$ ).

\section{Discussion}

The results from the belief in the conditional and acceptance of MP tasks replicated our findings from Experiment 1 . Although the role of disabling conditions as causes of the exceptions was emphasized by assigning different numbers of exceptions directly to them, there was no effect of number of disabling conditions. Participants seem to have evaluated their beliefs in the conditional and the conclusion of MP solely on the basis of frequency information.

Unlike in Experiment 1, the number of exceptions did not have an effect on MT in Experiment 2. We have no explanation for this failure to replicate. Moreover, the finding that a high number of disabling conditions led to inferring MT more willingly (even if only to a small extent) contradicts all of the previous findings of research on the suppression effect. Our findings also directly contradict the predictions of the support theory (Tversky \& Koehler, 1994). Unpacking the conditions that lead to a certain probability of exceptions did not lead to a decrease in the probability judgments of the conditional or reduce inference acceptance. Again, as in Experiment 1, there was no effect of any of the manipulations on $\mathrm{AC}$ and $\mathrm{DA}$, in line with previous literature on the necessity and sufficiency of conditionals.

The main question the results of Experiment 1 and 2 raise is whether the information about disabling conditions, if used without the frequency information on exceptions given in these experiments, would yield the expected result of diminishing belief in the conditional and suppressing the inferences of MP and MT. It could be claimed that the disabling conditions used in these experiments did not yield the usual suppression effect because the material consisted of artificial reasons for unfamiliar circumstances and thus did not work in general. To test this hypothesis, in a third experiment we presented participants with disabling conditions exclusively, omitting any frequency information about exceptional cases, and asked them again to rate their belief in the conditional and their confidence in the four inferences.

\section{EXPERIMENT 3}

\section{Method}

Participants. Participants comprised 22 students (age range: 20-27 years) from the University of Potsdam, majoring in various subjects. The order of tasks was again varied; one group of 11 participants first gave probability judgments and then solved reasoning tasks, and the other group of 11 performed these tasks in the reverse order.

Materials and Procedure. The experiment was a within-subjects study. The procedure was the same as the one used in Experiments 1 and 2. The material consisted of the same eight cover stories, but this time participants were not provided with any frequency information about exceptional cases. The number of disabling conditions used in Experiments 1 and 2 varied between 0, 1, 2, and 3. We tested all intermediate levels of disabling information in order to be able to compare our results to De Neys et al. (2003b). The only frequency information that was kept in the cover stories was the overall number of cases (i.e., items that were investigated); this was kept in order to provide participants with some anchor for their beliefs in the otherwise completely arbitrary conditionals. The selection of disabling conditions, when fewer than three were displayed, was randomized anew for each participant and conditional. Appendix B lists all of the disabling conditions used.

\section{Here is an example of a cover story for Condition 4.}

A team of biologists examines the different species on Noxus. They focus on genetic relationships expressed by body characteristics, such as number of legs and the shape of ears. They find that if an animal belongs to the family of grocks, then it has six legs. The biologists have examined 2,000 animals so far and have discovered that:

- If a grock has a genetic mutation, then it has fewer than six legs.

- If a grock is born to lop-eared parents, then it has fewer than six legs.

- If a grock has run into a trap for rat-like animals, then it has fewer than six legs

The presentation of the probability judgment questions and the four inference tasks was identical to that of Experiments 1 and 2 in form and order.

\section{Results}

Since the task order factor did not have a general effect and did not interact with any of the other factors, all data were collapsed over this factor and submitted to a one-factor (number of disabling conditions: 1, 2, 3, or 4) ANOVA.

Figure 1A shows the effect of number of disabling conditions on participants' evaluations of the believability of the conditional statement $[F(1,19)=35.2, p<.001$, $\left.\eta_{\mathrm{p}}^{2}=0.63\right]$. Planned contrasts revealed that any additional disablers, up to two, significantly lowered the believability ratings: zero versus one disabler $[F(1,21)=21.2, p<$ $.001]$ and one versus two disablers $[F(1,21)=12.0, p<$ $.01]$. Introducing a third disabler did not have an additional effect: two versus three disablers $(F<1)$.

Figure 1B shows the confidence ratings for all inferences. The number of disabling conditions had an overall effect on 
A Belief in the Conditional

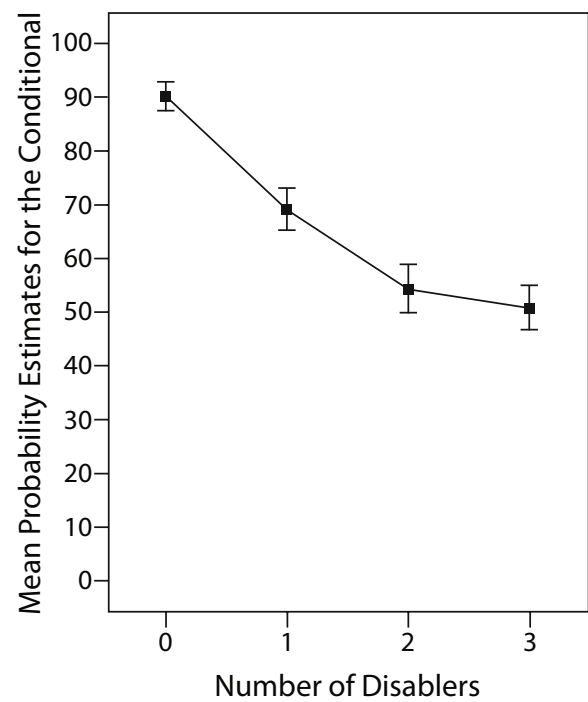

B Acceptance of Inferences

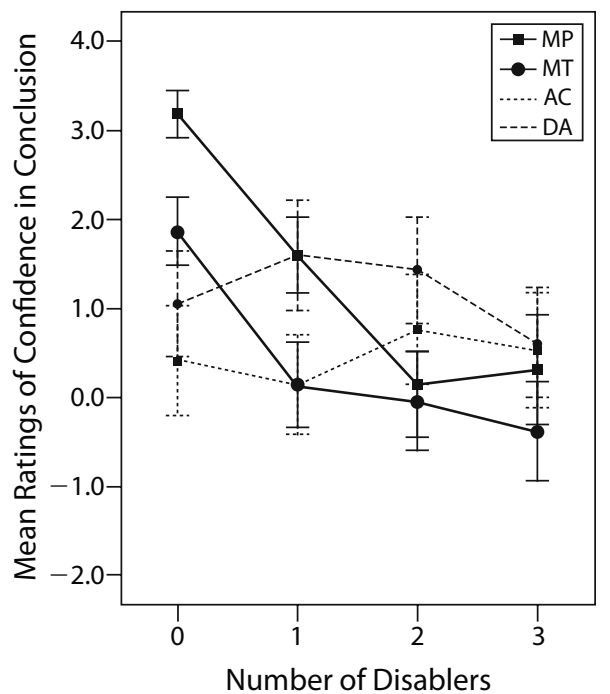

Figure 1. Panel A shows probability estimates (on a scale from 0 to 100) for the believability of the conditional statement in Experiment 3. Panel B shows confidence ratings (on a scale from -5 to +5 ) for the inference tasks. The numbers of disablers presented appear on the $x$-axis. Error bars depict the standard errors of the means.

MP $\left[F(1,19)=12.1, p<.001, \eta_{\mathrm{p}}^{2}=0.37\right]$ with additional effects for the first two disabler conditions: zero versus one disabler $[F(1,21)=11.1, p<.01]$, one versus two disablers $[F(1,21)=8.2, p<.01]$, and no additional effect for the third disabler condition, two versus three disablers $(F<1)$. There was also an overall effect of disabler on MT $[F(1,19)=5.0$, $\left.p<.01, \eta_{\mathrm{p}}^{2}=0.19\right]$. For MT only, the first disabler significantly lowered acceptance rates: zero versus one disabler $[F(1,21)=7.5, p<.05]$; the second and the third disablers did not additionally lower inference acceptance compared with the closest condition (both $F_{\mathrm{S}}<1$ ). Effects on $\mathrm{AC}$ and DA did not reach significance (all $F \mathrm{~s}<1.5$ ).

\section{Discussion}

In Experiment 3, we showed that the disabling information used in Experiments 1 and 2 did indeed have the expected suppressing effect on belief in the conditionals MP and MT when the conditionals were presented without information concerning the frequency of exceptions. This replicates the findings of De Neys et al. (2003b), who performed a similar manipulation of disabling information on inference tasks. The number of disablers varied between zero and four and concerned everyday content. We also found stronger effects for the first two disablers than for additional, later disablers on MP and MT, and we found no effects at all on AC and DA.

Moreover, with Experiment 3 we proved that the disabling information used in Experiments 1 and 2 was effective when used without information on the frequency of exceptions. The lack of effect of disabling conditions in these experiments cannot, therefore, be attributed to the fictional content of the material.

\section{EXPERIMENT 4}

In the fourth experiment, we tested whether our findings would extend to everyday conditionals. It could be that in the previous experiments, people used frequency information exclusively because it was explicitly given and therefore very easily available; this may have created a demand, within the tasks, to use frequency information. When frequency information is used, disabler information is rendered redundant. Using everyday conditionals allowed us to test a more natural situation in which both kinds of information had to be retrieved from memory. In such a situation, frequency information may not be as readily available, and people may draw on causal knowledge about disablers instead of, or in addition to, knowledge about frequencies. One could even speculate that frequency information is available in memory only through disabler information, because the frequency of exceptions is represented as the frequency (or probability) of the disabling conditions that cause them.

To test this hypothesis, we conducted a fourth experiment using conditionals with everyday content. In a pretest, 85 conditional statements were rated on the basis of their average number of exceptional cases and disabling conditions. We selected 20 conditionals on the basis of these ratings to fill the four cells of the design presented in Table 3.

\section{Method}

\section{Pretests}

A set of 85 conditionals with everyday content was constructed in a manner that insured a range of combinations of the two dimensions of interest. Two different groups of participants rated the conditionals in this set in two categories: the average number of exceptions to the conditional rule and possible disabling conditions. 
Table 3

Design Used in Experiment 4, With Example Items

\begin{tabular}{|c|c|c|}
\hline \multirow{2}{*}{$\begin{array}{l}\text { Exceptional } \\
\text { Cases }\end{array}$} & \multicolumn{2}{|c|}{ Disabling Conditions } \\
\hline & Few & Many \\
\hline Few & $\begin{array}{l}\mathrm{FF}: \text { If water is heated to } \\
100^{\circ} \mathrm{C} \text {, then it will boil. }\end{array}$ & $\begin{array}{l}\text { FM: If you open the fridge, } \\
\text { then the light inside goes on. }\end{array}$ \\
\hline Many & $\begin{array}{l}\text { MF: If a horse is white, } \\
\text { then it is an albino. }\end{array}$ & $\begin{array}{l}\text { MM: If you drink coffee in } \\
\text { the evening, then you won't } \\
\text { be able to fall asleep. }\end{array}$ \\
\hline
\end{tabular}

Note-The first letter of the condition code represents exceptional cases ( $p \neg q$ cases), and the second letter represents the number of disabling conditions (i.e., conditions that allow or cause $p \neg q$ cases).

PretestA: Exceptions. To ensure additivity of $P(p q)$ and $P(p \neg q)$, we asked for frequencies of $p q$ and $p \neg q$ cases. Twenty first-year university students answered the following questions about all 85 conditionals.

Consider the following statement: If you open the fridge, then the light goes on inside. Please imagine opening the fridge on 100 separate occasions. In your opinion, on how many of these 100 occasions, on average, does it happen that: (1) the light goes on inside? (2) the light does not go on inside? Please consider, of course, that the two numbers have to add up to 100. Estimate the frequency simply on the grounds of your everyday experience.

Examples of an answer scheme were given in the general instructions for this pretest and for Pretest B. As a control, we computed the sums of each participant's answers to both questions, and all sums equaled 100 .

Pretest B: Disabling conditions. Since the answer format for disabling conditions is more time consuming than the exceptions question, each participant received only 10 conditionals, and the survey was distributed on the Internet in order to recruit a sufficient number of participants. Two hundred participants answered the questions about disabling conditions for 10 conditionals randomly drawn from the total of 85 . Data produced by people who did not complete the whole questionnaire were nevertheless analyzed. The mean number of judgments for each conditional was $22.3(S D=4)$. The mean number of judgments made by each participant was 9.5 $(S D=1.5)$. The instructions were as follows.

Consider the following statement: If you open the fridge, then the light goes on inside. Can you imagine circumstances in which the following is possible: You open the fridge, and the light inside does not go on?

If yes, please list, in the table below [where six empty text input slots were provided], as many causes as you can think of for this situation. Please list all of the causes that you think are plausible simply on grounds of your everyday experience. If you cannot think of as many as six causes, list the ones you can think of and leave the remaining slots empty.

Two independent raters scored the responses and eliminated all that were judged to be unrealistic variations of one single idea.

On the basis of the two ratings, five conditionals were chosen to fit in each cell of the 2 (exceptions: few vs. many) $\times 2$ (disablers: few vs. many) design. Conditionals in each cell were chosen if their score was above the 60th percentile or below the 40th percentile line. Cell means were calibrated so that they were roughly an equal distance from the overall median for each dimension. For instance, the mean of the few disablers condition (1.2) and the mean of the many disablers condition (2.6) differed from the median rating of 1.9 by the same absolute amount. Ratings for the 20 conditionals used in Experiment 4 are shown in Appendix B.

\section{Main Experiment}

Participants. A total of 30 high school seniors and first-year university students, studying various subjects, participated in this experiment.
The order of tasks was varied among participants; one group of 15 first made probability judgments and then solved reasoning tasks, and the other 15 participants performed these tasks in the opposite order.

Materials and Procedure. The experiment was a within-subjects study that followed the design presented in Table 3 . The procedure that was used to assess the dependent measures was the same as the one used in Experiments 1-3.

\section{Results}

Since everyday conditionals can vary in other untested dimensions, we refrained from analyzing the answers for $\mathrm{AC}$ and DA. Variables with well-known influences on these inferences (mainly necessity and availability of alternative antecedents) have not been established for the conditionals used in Experiment 4. Data for the remaining three dependent variables (belief in the conditionals, MP, and MT) were submitted to $2 \times 2 \times 2$ ANOVAs with number of exceptions (few vs. many), number of disabling conditions (few vs. many), and task order (probability judgments first vs. reasoning tasks first) as factors. Unlike in the other three experiments, the latter factor did have an effect, and the results for belief in the conditionals and reasoning tasks are reported separately.

\section{Probability of the Conditional}

Figure 2 shows participants' evaluation of the believability of the conditional statements. Number of exceptions had a large effect $\left[F(1,28)=288.6, p<.001, \eta_{\mathrm{p}}^{2}=\right.$

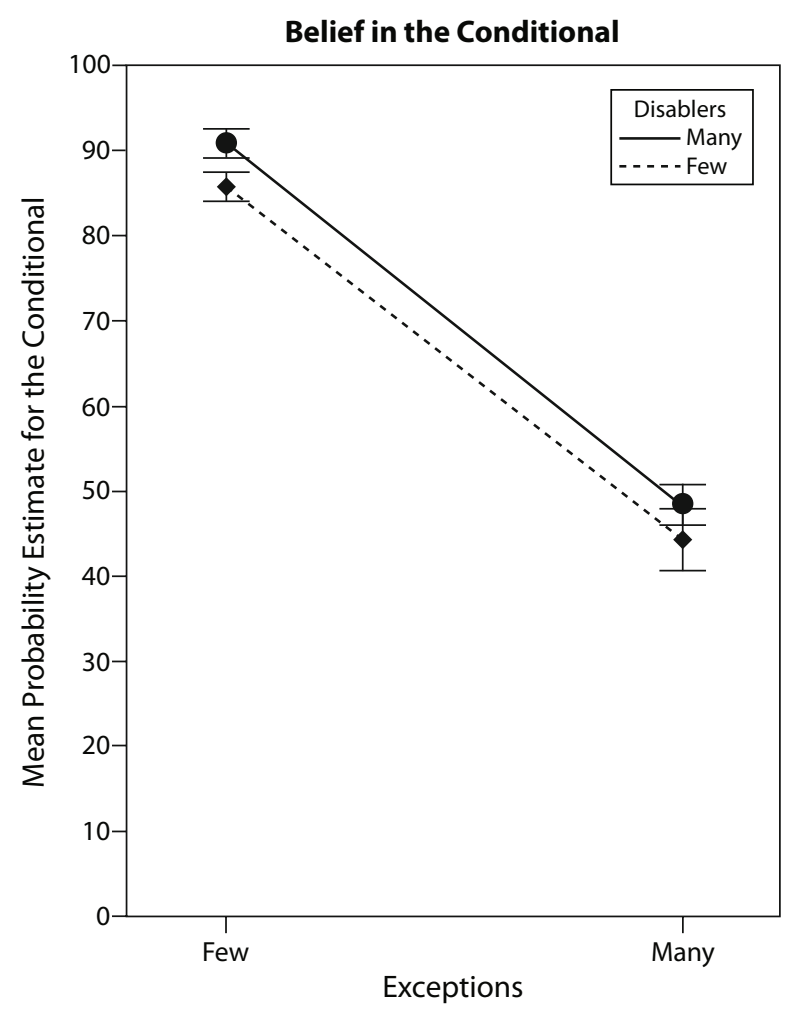

Figure 2. Probability estimates (on a scale from 0 to 100) for the believability of the conditional statement in Experiment 4. Numbers of exceptions appear on the $x$-axis; the different numbers of disablers are indicated by different line patterns. Error bars depict the standard errors of the means. 

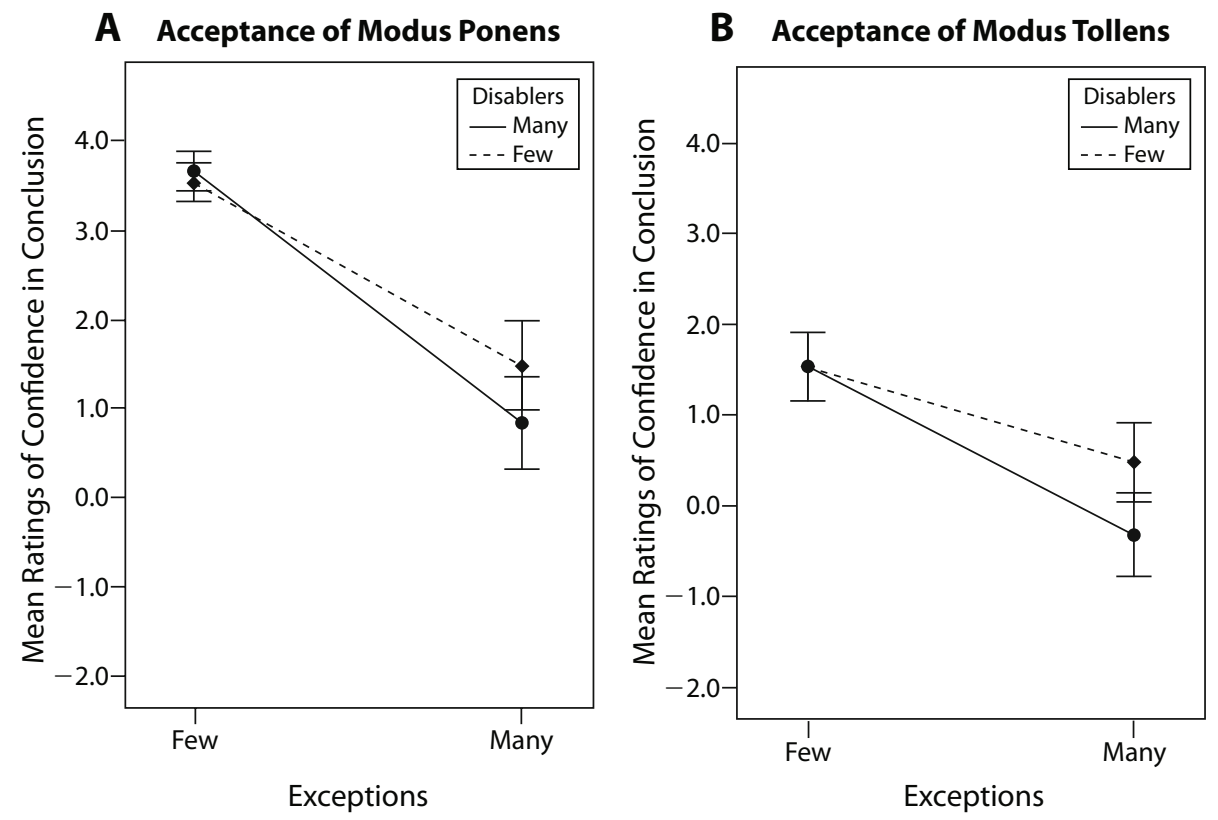

Figure 3. Confidence ratings in the modus ponens and modus tollens conclusions (on a scale from -5 to +5 ) in Experiment 4. Numbers of exceptions appear on the $x$-axis; the different numbers of disablers are indicated by different line patterns. Error bars depict the standard errors of the means.

0.91]. Number of disabling conditions had a smaller effect on the believability of the conditional $[F(1,28)=8.1, p<$ $\left..01, \eta_{\mathrm{p}}^{2}=0.22\right]$. Unexpectedly, conditionals with many disabling conditions were rated higher for believability than were conditionals with few disabling conditions. Task order also had a main effect on the believability of the conditionals. When participants performed the probability judgments first, the believability was rated lower $\left[F(1,28)=14.6, p=.01, \eta_{\mathrm{p}}^{2}=0.34\right]$.

Unlike in the previous experiments, task order interacted with both of the other factors. The influence of exceptions as well as of disabling conditions was higher when probability judgments were given first. There was a small interaction effect of task order and exceptions $[F(1,28)=7.3, p<.05$, $\left.\eta_{\mathrm{p}}^{2}=0.21\right]$, such that the suppression effect of exceptions was higher when the conditional was judged first. The interaction of task order and disabling conditions also reached significance $\left[F(1,28)=6.4, p<.05, \eta_{\mathrm{p}}^{2}=0.19\right]$, such that more disabling conditions led to higher believability ratings only when the conditional was judged first $[t(1,14)=$ $-3.18, p<.01]$. The interaction of all three factors was significant as well $\left[F(1,28)=6.3, p<.05, \eta_{\mathrm{p}}^{2}=0.18\right]$.

\section{Reasoning Tasks}

Figure 3A shows participants' confidence in MP. Number of exceptions again had the largest effect $[F(1,28)=$ $\left.42.2, p<.001, \eta_{\mathrm{p}}^{2}=0.60\right]$. There was a nonsignificant trend for MP to be endorsed more when fewer disablers were available $\left[F(1,28)=3.2, p=.09, \eta_{\mathrm{p}}^{2}=0.10\right]$. For the interaction of both factors, we also observed a trend that just missed the conventional criterion of significance $\left[F(1,28)=3.2, p=.06, \eta_{\mathrm{p}}^{2}=0.12\right]$. Number of disablers seemed to have a suppressing effect only when the number of exceptions was high.

Task order had a main effect on MP: When probability judgments were given first, MP was endorsed more hesitantly $\left[F(1,28)=4.4, p<.05, \eta_{\mathrm{p}}^{2}=0.14\right]$. Moreover, task order interacted with number of disabling conditions $[F(1,28)=$ $\left.6.8, p<.05, \eta_{\mathrm{p}}^{2}=0.20\right]$; disabling conditions had a suppressing effect only when inferences were evaluated first.

Figure 3B shows participants' confidence in MT. Number of exceptions again had the largest effect on this inference $\left[F(1,28)=31.0, p<.001, \eta_{\mathrm{p}}^{2}=0.53\right]$. Number of disabling conditions did not have a general effect on MT $[F(1,28)=2.8, p>.1]$ but interacted with task order $\left[F(1,28)=8.2, p<.01, \eta_{\mathrm{p}}^{2}=0.23\right]$. A suppressing effect of disabling conditions showed only in the group that evaluated inferences first $[t(1,14)=2.76, p<.05]$. There was no effect in the group that judged the conditionals first $[t(1,14)=-1.0, p=.3]$. None of the other factors reached significance (all $\left.F_{\mathrm{S}}<1.6\right)$.

\section{Discussion}

Experiment 4 orthogonally varied the number of disabling conditions and the relative frequency of exceptions, drawing on people's knowledge about conditionals with everyday contents. In the main experiment, both kinds of information (disablers and frequency of exceptions) had to be retrieved from memory; by thus presenting information in the task context, any demand characteristic was avoided. People could base their judgments about the believability of the conditionals and about the inferences that could be drawn from them on one type of information or the other, or on a combination of both. 
As in the previous experiments, in evaluating their beliefs in the conditional statements and their confidence in MP and MT, participants strongly favored the frequency of exceptional cases over the number of different disabling conditions. Hence, the large suppressing effect of frequency of exceptions on these three variables was replicated. Frequency information seems to have a dominating role in evaluating and reasoning with everyday conditionals, just as it did in reasoning with the arbitrary conditionals that were used in the probabilistic truth table tasks in Experiments 1 and 2.

Number of disabling conditions exerted only a minor effect on belief in the conditionals; contrary to expectations, belief was higher when more disabling conditions were available. This paradoxical effect might be due to specific characteristics of the 20 conditional sentences used in Experiment 4, which may have had an untested impact on the believability of those sentences. For MP and MT, disabling conditions only showed a trend toward a suppression effect; in the case of MT, this trend was apparent only when reasoning tasks were evaluated first. Taking these results together, it seems justified to assume that for everyday conditionals as much as for arbitrary conditionals, people rely primarily on frequency information when evaluating the believability of a conditional statement and the MP and MT inferences drawn from that conditional.

This main effect was modulated by several small interaction effects of the experimental manipulations with task order. When the believability judgment for the conditional was made first, the number of exceptions had a larger effect than when this judgment was made second. On the other hand, when reasoning tasks were solved first, number of disabling conditions had a larger effect (i.e., a small effect as compared with no effect at all) on acceptance of MP and MT.

The present results seem to contradict those from Weidenfeld et al.'s (2005) study. These authors investigated correlations across conditionals among the number of disabling conditions people could think of, their degree of belief in conditional statements, their estimates of $P(q \mid p)$, and endorsement rates for MP and MT. For the inductive inferences, which are most comparable with the instructions used in Experiment 4, Weidenfeld and colleagues found that the number of disabling conditions had an equally strong direct effect on the rates of endorsement for MP and MT as did the belief in the conditional and $P(q \mid p)$.

In the present study, we found a different overall pattern of effects. Across the 20 conditionals, mean subjective $P(q \mid p)$ values (calculated from the ratings of the frequency of $q$ given $p$ ) strongly correlated with mean endorsement of MP $(r=.90, p<.01)$ and MT $(r=.59$, $p<.01)$, whereas the number of disabling conditions was not correlated with either MP or MT $(p>.18)$. Since in the Weidenfeld et al. (2005) study, participants who rated the inferences did not give any other ratings at all, Weidenfeld et al.'s results are best compared with the results from our group that rated the inferences first. In that group, we found a small but significant suppression effect of the number of disabling conditions for both MP and MT. The discrepancy between the present findings and those of Weidenfeld et al. is therefore more quantita- tive than qualitative: Whereas they found that disabling condition and $P(q \mid p)$ had equally strong effects on inference endorsement, we found disabling conditions to have a smaller effect than $P(q \mid p)$.

This discrepancy may be due to methodological differences in the two studies. Weidenfeld et al. (2005) used pseudonaturalistic conditionals with fictional contents that could be related only indirectly to world knowledge, whereas we used conditionals that derived directly from people's knowledge. Moreover, Weidenfeld et al. made no effort to deconfound $P(q \mid p)$ and the number of disabling conditions, so the stronger effect of disabling conditions in their study might be the result of confounds with the $P(q \mid p)$ measure. A third difference regards the response format for the inference tasks. Weidenfeld et al. asked participants to make a categorical judgment either accepting or rejecting the conclusion, whereas we asked our participants to rate their confidence in the conclusion on a continuous scale. The continuous scale could have motivated participants to frame the task as one of probability estimation, for which it seems rational to search for information about the relative frequencies of cases in which the conclusion holds. However, when the task is to decide whether a conclusion can or cannot be drawn from a set of premises, it seems rational to search for cases that refute the conclusion - that is, disabling conditions.

\section{GENERAL DISCUSSION}

There is ample evidence that the availability of counterexample information suppresses otherwise endorsed inferences (Byrne, 1989; Byrne et al., 1999; Cummins, 1995; De Neys, Schaeken, \& d'Ydewalle, 2003a, 2003b; Quinn $\&$ Markovits, 1998) and lowers the perceived sufficiency of a conditional sentence (Dieussaert et al., 2002). This counterexample information is usually conceptualized as causal information that expresses a condition or factor that prevents the consequent of a conditional statement from occurring even though the antecedent is given.

Another, more recent approach to the understanding of conditionals advocates a probabilistic view, according to which conditionals are evaluated by comparing ruleconfirming instances of the $p q$ conjunction with exceptional instances of the $p \neg q$ conjunction (Evans \& Over, 2004; Evans et al., 2005; Oberauer et al., 2007). In this line of research, a probabilistic truth table task has been established that presents explicit frequency information about exceptional cases. One of the major findings with this paradigm is that the belief in a conditional is highly dependent on the ratio of $p q$ to $p \neg q$ cases. A corresponding effect of explicit frequency information on the inference tasks MP and MT was demonstrated in a recent study (Geiger \& Oberauer, 2006).

Verschueren et al. (2005) have systematically investigated these two different types of information and have found that frequency information as well as counterexample information that can be retrieved from memory exert a suppressing effect on inferences, depending on the speed of judgments and contextual factors (e.g., the association strength of counterexamples). 
The present experiments took a similar approach. Experiments 1 and 2 combined explicitly mentioned frequency information (as used in the probabilistic approach) with explicitly mentioned counterexample information (as used in studies on suppression effects). In both experiments, the effect of frequency information about exceptions outweighed the influence of information about categories of disablers. Experiment 3 established that this result was not due to specificities of the materials used in Experiments 1 and 2 . The fourth experiment largely replicated the findings of the first two experiments, using a carefully selected set of everyday conditionals across which the number of disablers and the frequency of exceptional cases was varied orthogonally. This independent manipulation is important since the two dimensions are usually highly correlated (i.e., usually, the more disabling conditions there are to prevent $q$ in the presence of $p$, the more exceptional $p \neg q$ cases there are). By choosing sentences that varied independently on these two dimensions, we were able to isolate the effects of any one dimension. The results unambiguously show that people give priority to information about the frequency of exceptions over causal information about the number of different disabling conditions.

Why do people prefer frequency information over disabling conditions, which could be more informative (e.g., by giving us reasons for exceptions and pointing toward similar circumstances in which to expect exceptions)? In a probabilistic framework, relying exclusively on frequency information is rational. According to this view, the probability of the conditional is a function of $P(q \mid p)$, which in turn depends on the relative frequency of exceptions, regardless of the number of disabling conditions by which these exceptions were caused. MP and MT in turn depend on the believability of the major conditional premise and are therefore likewise affected by the relative frequencies of exceptions. In a probabilistic framework, there is no reason to take the number of disabling conditions into account if the believability of a conditional can be evaluated via frequency information. Previous findings have shown that endorsement of inferences from conditionals is blocked by the availability of disabling conditions (see, e.g., Cummins, 1995; De Neys et al., 2002, 2003b), but this blocking is probably mediated by people's beliefs about the relative frequencies of exceptions: In nonselected samples of everyday conditionals, people's beliefs about the relative frequency of exceptions are highly correlated with the availability of disabling conditions. Thus, in the absence of independent information about the frequency of exceptions, the number of different possible causes of such exceptions is a good estimate of their probability of occurrence.

The present results have implications for theories of how people reason from conditional premises. It has been well documented that content and context modulate people's readiness to accept even the logically valid inferences MP and MT. In the mental model framework, this so-called inference suppression effect has been explained by assuming that people can retrieve models of counterexamples to the conditional premise - that is, models of the $p \neg q$ conjunction. According to authors working in the context of the mental model theory (e.g., Klaczynski,
2001; Markovits \& Quinn, 2002), retrieving a single such model should be sufficient to block endorsement of MP and MT, because if $p \neg q$ is represented as a possibility, it constitutes a counterexample to the conclusions of MP and MT, and hence these conclusions are not supported by the set of mental models of the situation that the premises describe. Against this assumption, De Neys et al. (2003b) have shown that people's degree of endorsement of MP and MT declines linearly with every additional counterexample that is presented to them or that they can retrieve from the conditional premise in a separate part of the experiment.

Our results add further evidence against the mental models account of the inference suppression effect. Whether a mental model of the $p \neg q$ conjunction is constructed should depend on whether people think of that conjunction as a possibility, not on how frequently people believe this conjunction occurs. The theory of mental models allows for attaching numerical information about frequencies or probabilities to mental models (JohnsonLaird, Legrenzi, Girotto, Legrenzi, \& Caverni, 1999), but this numerical information plays a role only in probability estimation tasks, not in conditional inferences. The only way in which the mental model theory could explain why beliefs about the frequency of exceptions affect endorsement of MP and MT is by framing these inference tasks as probability estimation tasks. A probabilistic model theory of reasoning with conditionals could assume that people assign probabilities to each mental model and estimate the probability of the conclusion from these values. For instance, in an MP argument, the minor premise, $p$, serves to reduce the set of models to those involving $p$ - that is, the $p q$ model and the $p \neg q$ model. People might assign probability .8 to the $p q$ model and probability .2 to the $p \neg q$ model, and from that they might infer a degree of belief in the conclusion, $q$, of .8. A probabilistic adaptation of the model theory is not implausible in light of the fact that we asked participants to evaluate conclusions on a continuous scale rather than to make categorical judgments of acceptance or rejection. Of course, such an adaptation would bring the model theory very close to probabilistic theories of reasoning with conditionals.

Our findings are consistent with probabilistic theories of the interpretation of and reasoning from conditionals. These theories agree that people's degree of belief in a conditional is determined by the subjective conditional probability of the consequent, given the antecedent. The effect of the relative frequency of exceptions on belief in the conditional confirms this assumption.

Regarding inferences people draw from conditionals, probabilistic theories differ in their assumptions. Evans and Over (2004) assumed that endorsement of MP and MT depended on people's degree of belief in the conditional premise. Oaksford et al. (2000) assumed instead that people evaluated the conditional probability of the conclusion, given the minor premise. This reasoning process sidesteps an evaluation of the conditional premise. The present data do not distinguish between these two views - both predict that, in the present design, the relative frequency of exceptions should have influenced how 
readily people endorsed MP and MT, and our findings confirmed this prediction (for a test of predictions that distinguish the two probabilistic views, see Geiger \& Oberauer, 2006).

One theory that attempts to reconcile the mental model framework with probabilistic theories is the dual process account of Verschueren et al. (2005). According to this view, inferences from conditional premises are evaluated through two processes. A fast, heuristic process draws on knowledge about the frequency or probability of exceptions to assess the believability of the conditional and evaluates the conclusion accordingly. A slower, analytical process draws on causal knowledge about disabling conditions and operates according to the assumptions of the mental model theory (Markovits \& Barrouillet, 2002; Schroyens, Schaeken, \& Handley, 2003). The dual process theory predicts that fast judgments on conditional inferences will be affected mostly by frequency information and slow judgments will be affected mostly by information about disabling conditions. In our experiments, participants made their judgments without time pressure, so our results should reflect a mixture of fast and slow judgments. Verschueren et al.'s theory would, therefore, predict that both the frequency of exceptions and the number of disabling conditions would have had effects on people's endorsements of MP and MT. This was not what we found. The dual process account of Verschueren et al. could explain our data only with the additional assumption that participants in our experiments relied nearly exclusively on the fast, heuristic process. With this assumption, however, the theory reduces to a purely probabilistic one.

Such a purely probabilistic view of inferences from conditionals, which is most compatible with the present data, could be challenged by other findings, however. De Neys et al. (2002) found that people took more time to make judgments on MP inferences when there were more counterexamples and interpreted this finding as reflecting the time needed to retrieve the counterexamples. If judgments on inferences were based only on a process that accesses frequency information quickly and automatically, no such effect would be expected. De Neys, Schaeken, and d'Ydewalle (2005) observed an interaction of working memory capacity and secondary task load on inference acceptance, which they interpreted as reflecting the demands of working memory on retrieving counterexamples and on evaluating their relevance. No such effects would be expected if inference evaluation were based only on automatic retrieval of frequency information. There is nothing in our data, however, that implies that frequency information is retrieved automatically and without demands on working memory. The effects that De Neys and colleagues attributed to retrieval of counterexamples could be due to retrieval of frequency information instead.

More problematic for a purely probabilistic view are the results of a series of tests of formal models applied to patterns of acceptance or rejection of the four basic inferences from conditionals (Oberauer, 2006). Whereas the probabilistic model of Oaksford et al. (2000) gave a poor account of the data, a variant of the mental model theory and the dual process model of Verschueren et al.
(2005) fit the data well. Unlike the data from the present study, the data modeled by Oberauer (2005) consisted of categorical judgments of acceptance or rejection of the four inferences. As we argued above in the Discussion of Experiment 4, the response format - continuous estimates of confidence in a conclusion versus categorical judgments - could induce different modes of reasoning, with the former giving rise to probability estimation and the latter to model-based reasoning. A direct comparison of the two response formats could help to further clarify the roles of probabilistic and model-based reasoning in people's evaluation of inferences from conditionals.

\section{CONCLUSION}

In four experiments, we showed that people fix their belief in a conditional and their confidence in conclusions drawn from it on the basis of the frequency of cases that confirm the condition relative to the frequency of exceptions to it. The number of disabling cases that cause these exceptions played a minor role in inference acceptance and was noticeable only when inference judgments preceded believability judgments of the conditional. The finding that frequency of exceptions was the main factor determining acceptance of MP and MT is consistent with probabilistic theories of conditional reasoning but seriously challenges the current formulation of the mental model theory. In our study, there was little evidence for an additional search for counterexamples above and beyond probabilistic estimations (at least with the gradual answer scale we used for evaluating the inferences), which calls into question the necessity of the analytic process assumed by dual process theories of reasoning.

\section{AUTHOR NOTE}

This research was supported by Deutsche Forschungsgemeinschaft Grant FOR 375 1-1. We thank Petra Grüttner for testing the participants of Experiments 2 and 4 and Niki Verschueren for providing many of the conditionals used in Experiment 4. We also thank Wim De Neys, Berry Claus, and Annekatrin Hudjetz for fruitful comments on the work and on earlier drafts of this paper. Correspondence concerning this article should be addressed to S. M. Geiger, Department of Psychology, P.O. Box 601553, 14415 Potsdam, Germany (e-mail: geiger@cyllene.uwa.edu.au).

\section{REFERENCES}

Anderson, J. R. (1995). Cognitive psychology and its implications. New York: Freeman.

BYRne, R. M. (1989). Suppressing valid inferences with conditionals. Cognition, 31, 61-83.

Byrne, R. M., EsPino, O., \& Santamaria, C. (1999). Counterexamples and the suppression of interferences. Journal of Memory \& Language, 40, 347-373.

Cummins, D. D. (1995). Naive theories and causal deduction. Memory \& Cognition, 23, 646-658.

Cummins, D. D., Lubart, T., Alksnis, O., \& Rist, R. (1991). Conditional reasoning and causation. Memory \& Cognition, 19, 274-282.

De Neys, W., Schaeken, W., \& D'Ydewalle, G. (2002). Causal conditional reasoning and semantic memory retrieval: A test of the semantic memory framework. Memory \& Cognition, 30, 908-920.

De Neys, W., Schaeken, W., \& D’Ydewalle, G. (2003a). Causal conditional reasoning and strength of association: The disabling condition case. European Journal of Cognitive Psychology, 15, 162-167.

De Neys, W., Schaeken, W., \& D'Ydewalle, G. (2003b). Inference 
suppression and semantic memory retrieval: Every counterexample counts. Memory \& Cognition, 31, 581-595.

De Neys, W., Schaeken, W., \& D'Ydewalle, G. (2005). Working memory and everyday conditional reasoning: Retrieval and inhibition of stored counterexamples. Thinking \& Reasoning, 11, 349-381.

Dieussaert, K., Schaeken, W., \& D'Ydewalle, G. (2002). The relative contribution of content and context factors on the interpretation of conditionals. Experimental Psychology, 49, 181-195.

Evans, J. St. B. T., Handley, S. J., \& Over, D. E. (2003). Conditionals and conditional probability. Journal of Experimental Psychology: Learning, Memory, \& Cognition, 29, 321-335.

Evans, J. St. B. T., \& Over, D. E. (2004). If. Oxford: Oxford University Press.

Evans, J. St. B. T., Over, D. E., \& Handley, S. J. (2005). Suppositionals, extensionality, and conditionals: A critique of the mental model theory of Johnson-Laird and Byrne (2002). Psychological Review, 112, 1040-1052.

GeIger, S. M., \& Oberauer, K. (2006). How necessary is sufficiency for reasoning? A test of two probabilistic theories. Manuscript submitted for publication.

Johnson-LaIRD, P. N. (2001). Mental models and deduction. Trends in Cognitive Sciences, 5, 434-442.

Johnson-Laird, P. N., Legrenzi, P., Girotto, V., Legrenzi, M. S., \& CAVERNI, J.-P. (1999). Naive probability: A mental model theory of extensional reasoning. Psychological Review, 106, 62-88.

KLACZYNSKI, P. A. (2001). Analytic and heuristic processing influences on adolescent reasoning and decision making. Child Development, 72, 844-871

Markovits, H., \& Barrouillet, P. (2002). The development of conditional reasoning: A mental model account. Developmental Review, 22, 5-36.

Markovits, H., \& Potvin, F. (2001). Suppression of valid inferences and knowledge structures: The curious effect of producing alternative antecedents on reasoning with causal conditionals. Memory \& Cognition, 29, 736-744.

Markovits, H., \& QuinN, S. (2002). Efficiency of retrieval correlates with "logical" reasoning from causal conditional premises. Memory \& Cognition, 30, 696-706.

OAKSFORD, M., \& Chater, N. (1994). A rational analysis of the selection task as optimal data selection. Psychological Review, 101, 608-631.

OAKSFORD, M., \& ChATER, N. (2001). The probabilistic approach to human reasoning. Trends in Cognitive Sciences, 5, 349-357.
OAksford, M., Chater, N., \& Larkin, J. (2000). Probabilities and polarity biases in conditional inference. Journal of Experimented Psychology: Learning, Memory, \& Cognition, 2B, 883-899.

Oberauer, K. (2006). Reasoning with conditionals: A test of formal models of four theories. Cognitive Psychology, 53, 238-283.

Oberauer, K., Geiger, S. M., Fischer, K., \& Weidenfeld, A. (2007). Two meanings of "if"? Individual differences in the interpretation of conditionals. Quarterly Journal of Experimental Psychology, 60, 790-819.

Oberauer, K., \& Wilhelm, O. (2003). The meaning(s) of conditionals: Conditional probabilities, mental models, and personal utilities. Journal of Experimental Psychology: Learning, Memory, \& Cognition, 29, 680-693.

Over, D. E., \& Evans, J. St. B. T. (2003). The probability of conditionals: The psychological evidence. Mind \& Language, 18, 340-358.

QuinN, S., \& MARKovits, H. (1998). Conditional reasoning, causality, and the structure of semantic memory: Strength of association as a predictive factor for content effects. Cognition, 68, B93-B101.

RotTenstreich, Y., \& TVersky, A. (1997). Unpacking, repacking, and anchoring: Advances in support theory. Psychological Review, 104, 406-415.

Schroyens, W., Schaeken, W., \& Handley, S. (2003). In search of counter-examples: Deductive rationality in human reasoning. Quarterly Journal of Experimental Psychology, 56A, 1129-1145.

THOMPson, V. (1995). Conditional reasoning: The necessary and sufficient conditions. Canadian Journal of Experimental Psychology, 40, 1-60.

Thompson, V. (2000). Task specific nature of domain general reasoning. Cognition, 76, 209-268.

Tversky, A., \& Koehler, D. J. (1994). Support theory: A nonextensional representation of subjective probability. Psychological Review, 101, 547-567.

Verschueren, N., Schaeken, W., \& D'Ydewalle, G. (2005). A dualprocess specification of causal conditional reasoning. Thinking \& Reasoning, 11, 239-278.

WEIDENFELD, A. (2004). Interpretation of and reasoning with conditionals: Probabilities, mental models, and causality. Unpublished doctoral dissertation, University of Potsdam.

Weidenfeld, A., Oberauer, K., \& Hörnig, R. (2005). Causal and noncausal conditionals: An integrated model of interpretation and reasoning. Quarterly Journal of Experimental Psychology, 58A, 1479-1513. 


\section{Conditional Statements and Their Disabling Conditions Used in Experiments 1-3}

1. If a flopper has Xathylen in its blood, then it suffers from Midosis. But, if a flopper has an additional substance (Xalsosan) in its blood, then it doesn't suffer from Midosis. If a flopper has a genetic mutation that makes it immune, then it doesn't suffer from Midosis. If a flopper develops antigens, then it suffers from Midosis.

2. If the probe is warmer than $22^{\circ} \mathrm{C}$, then it is rich in philoben gas. But, if the probe was sealed under enormous pressure, then it is not rich in philoben gas. If the container has a leak, then the probe is not rich in philoben gas. If the probe is stored for a long time before it is examined, then it is not rich in philoben gas.

3. If it thardons, then the streets get sticky (cf. Cummins, 1995). But, if the atmosphere holds an additional substance (K gas), then the streets do not get sticky. If one of the rare cleaning vehicles is in use, then the streets do not get sticky. If a powdery substance (Kalgoren) is strewn in advance, then the streets do not get sticky.

4. If the box is sealed, then it glows in the dark. But, if the box is forced open, then it does not glow in the dark. If the box has a permeable spot, then it does not glow in the dark. If the box is smaller than a match box, then it does not glow in the dark.

5. If an animal belongs to the family of grocks, then it has six legs. But, if the grock has a genetic defect, then it does not have six legs. If the grock has lop-eared parents, then it does not have six legs. If the grock has run into a trap for rat-like animals, then it does not have six legs.

6. If the tree-like plant has a square trunk, then it has purple leaves. But, if the wind is very heavy, then the tree-like plant does not have purple leaves. If the tree-like plant is more than 300 years old, then it does not have purple leaves. If the tree-like plant is located in soil that is rich in Krenalon, then it does not have purple leaves.

7. If the Karun roots have a striped pattern, then they contain valuable nutrients. But, if the Karun roots are harvested in midnight light, then they do not contain valuable nutrients. If the Karun roots are stored together with another vegetable-like plant called Ertonnel, then they do not contain valuable nutrients. If the Karun roots are stored too long, then they do not contain valuable nutrients.

8. If the flight objects have invisible wings, then they have more than two jet propulsions. But, if the flight objects have a turbo engine, then they do not have more than two jet propulsions. If the flight objects were intended for regional transport, then they do not have more than two jet propulsions. If the flight objects are from the first generation, then they do not have more than two jet propulsions. 


\section{APPENDIX B
Conditional Statements Used in Experiment 4}

Exceptions are measures of the average number, in percentages, of $p \neg q$ cases per $100 p$ cases; disablers are absolute numbers of reasons for $p \neg q$ cases people could think of (with a maximum of six).

Conditional Statement

Exceptions Disablers

Category 1

FF: Few Exceptions, Few Disablers

1. If water is heated to $100^{\circ} \mathrm{C}$, then it will boil.

2. If a dog has fleas, then it will scratch itself.

3 . If you cut your finger, then it will bleed.

4. If somebody is a politician, then his diary is full.

5. If somebody only buys a newspaper, then he pays cash.

Category 2

FM: Few Exceptions, Many Disablers

6. If you phone someone, then his telephone will ring.

7. If you depress the brakes, then the car will slow down.

8. If you open the fridge, then the light goes on inside.

9. If the light switch is turned, then the light will go on.

10. If you switch on your mobile, then you see something on the display.

$\begin{array}{rrr} & 6.1 & 0.91 \\ & 3.5 & 0.58 \\ & 6.0 & 1.15 \\ & 14.2 & 1.63 \\ \text { Mean } & 6.8 & 1.41 \\ & 7.3 & 1.13\end{array}$

\section{Category 3}

MF: Many Exceptions, Few Disablers

11. If you unplug the computer, then it will shut down. 37.0

12. If a horse is white, then it is an albino.

13. If a pet performs stunts, then it is a dog.

14. If a pullover is made of cashmere, then it has to be brought to a dry cleaner.

15. If a letter arrives without an address, then a carrier pigeon has brought it.

$\begin{array}{rrr} & 5.1 & 2.60 \\ & 13.2 & 2.21 \\ & 9.3 & 2.75 \\ & 9.4 & 2.86 \\ \text { Mean } & 5.4 & 2.42 \\ & 8.5 & 2.57\end{array}$

Category 4

MM: Many Exceptions, Many Disablers

16. If you park your car on an illegal spot, then you will get a parking ticket.

17. If someone stays in the cold for a long time, then he will get sick.

18. If a woman has sexual intercourse, then she will get pregnant.

19. If you drink a lot of coke, then you will get fat.

20. If you drink coffee in the evening, then you won't be able to fall asleep. 